Review

\title{
Interferon Induced IFIT Family Genes in Host Antiviral Defense
}

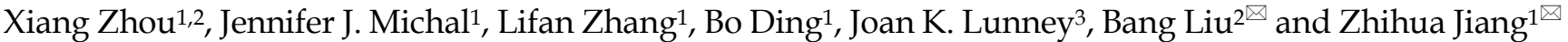 \\ 1. Department of Animal Sciences, Washington State University, Pullman, WA 99164-6351, USA \\ 2. Ministry of Education's Key Laboratory for Agricultural Animal Genetics, Breeding and Reproduction, and Ministry of Agriculture's \\ Key Laboratory for Swine Genetics and Breeding, College of Animal Science and Technology, Huazhong Agricultural University, Wu- \\ han 430070, Hubei, PR China \\ 3. Animal Parasitic Diseases Laboratory, ANRI, ARS, USDA, BARC-East, Beltsville, MD 20705, USA
}

$\triangle$ Corresponding author: Zhihua Jiang, Tel: +509 335 8761; Fax: +509 335 4246; E-mail: jiangz@wsu.edu and Bang Liu, Tel: +86 2787382290 ; Fax: +86 27 87280408; Email: liubang@mail.hzau.edu.cn.

(C) Ivyspring International Publisher. This is an open-access article distributed under the terms of the Creative Commons License (http://creativecommons.org/ licenses/by-nc-nd/3.0/). Reproduction is permitted for personal, noncommercial use, provided that the article is in whole, unmodified, and properly cited.

Received: 2012.11.27; Accepted: 2013.01.23; Published: 2013.02.1I

\begin{abstract}
Secretion of interferons (IFNs) from virus-infected cells is a hallmark of host antiviral immunity and in fact, IFNs exert their antiviral activities through the induction of antiviral proteins. The IFN-induced protein with tetratricopeptide repeats (IFITs) family is among hundreds of IFN-stimulated genes. This family contains a cluster of duplicated loci. Most mammals have IFITI, IFIT2, IFIT3 and IFIT5; however, bird, marsupial, frog and fish have only IFIT5. Regardless of species, IFIT5 is always adjacent to SLCI6AI2. IFIT family genes are predominantly induced by type I and type III interferons and are regulated by the pattern recognition and the JAK-STAT signaling pathway. IFIT family proteins are involved in many processes in response to viral infection. However, some viruses can escape the antiviral functions of the IFIT family by suppressing IFIT family genes expression or methylation of 5' cap of viral molecules. In addition, the variants of IFIT family genes could significantly influence the outcome of hepatitis C virus (HCV) therapy. We believe that our current review provides a comprehensive picture for the community to understand the structure and function of IFIT family genes in response to pathogens in human, as well as in animals.
\end{abstract}

Key words: IFIT family, evolution, antiviral activities, regulation and signaling, therapy of infectious diseases.

\section{Introduction}

Interferons (IFNs) are a family of proteins secreted by host cells in response to various pathogens such as viruses, bacteria, fungi, or parasites, which trigger the protective defenses of the immune system [1]. There are three types of IFNs in host animals: type I (IFN- $\alpha$, IFN- $\beta$ and IFN- $\omega)$, type II (IFN- $\gamma)$, and type III (IFN- $\lambda 1$, IFN- $\lambda 2$ and IFN- $\lambda 3$ ). All IFNs are secreted ligands of specific cell surface receptors that elicit the expression of hundreds of interferon stimulated genes (ISGs) [2-3]. Among them, the interferon-induced protein with tetratricopeptide repeats (IFITs) family has been heavily studied. Basically, this family of proteins is characterized by multiple repeats of tetratricopeptide repeat helix-turn-helix motifs that mediate a variety of protein-protein interactions involved in translation initiation, virus replication, double-stranded RNA signaling, cell migration, and proliferation [4]. Here we review the family's evolutionary features, expression patterns, antivirus activities, and genetic variants. Understanding the structure and function of IFIT family genes will certainly help elucidate how the immune system combats pathogens, 
thus improving therapy of infectious diseases in human, as well as in animals.

\section{IFIT Family Genes and Evolution}

Research has shown that the IFIT gene family is conserved in mammals, amphibians and fish, but does not exist in lower animals, like Drosophila melanogaster (fruit fly), Caenorhabditis elegans (nematode) and Saccharomyces cerevisiae (yeast), or in plants [5-6]. Based on the current reference genome assemblies, we collected data on the gene family in Homo sapiens (human), Macaca mulatta (rhesus monkey), Callithrix jacchus (common marmoset), Pongo abelii (sumatran orangutan), Canis familiaris (dog), Sus scrofa (pig), Bos taurus (cattle), Equus caballus (horse), Nomascus leucogenys (northern white-cheeked gibbon), Mus musculus (mouse), Rattus norvegicus (rat), Monodelphis domestica (gray short-tailed opossum), Gallus gallus (chicken), Xenopus (Silurana) tropicalis (western clawed frog) and Danio rerio (zebrafish). The first eight mammals have four members in the IFIT gene family:
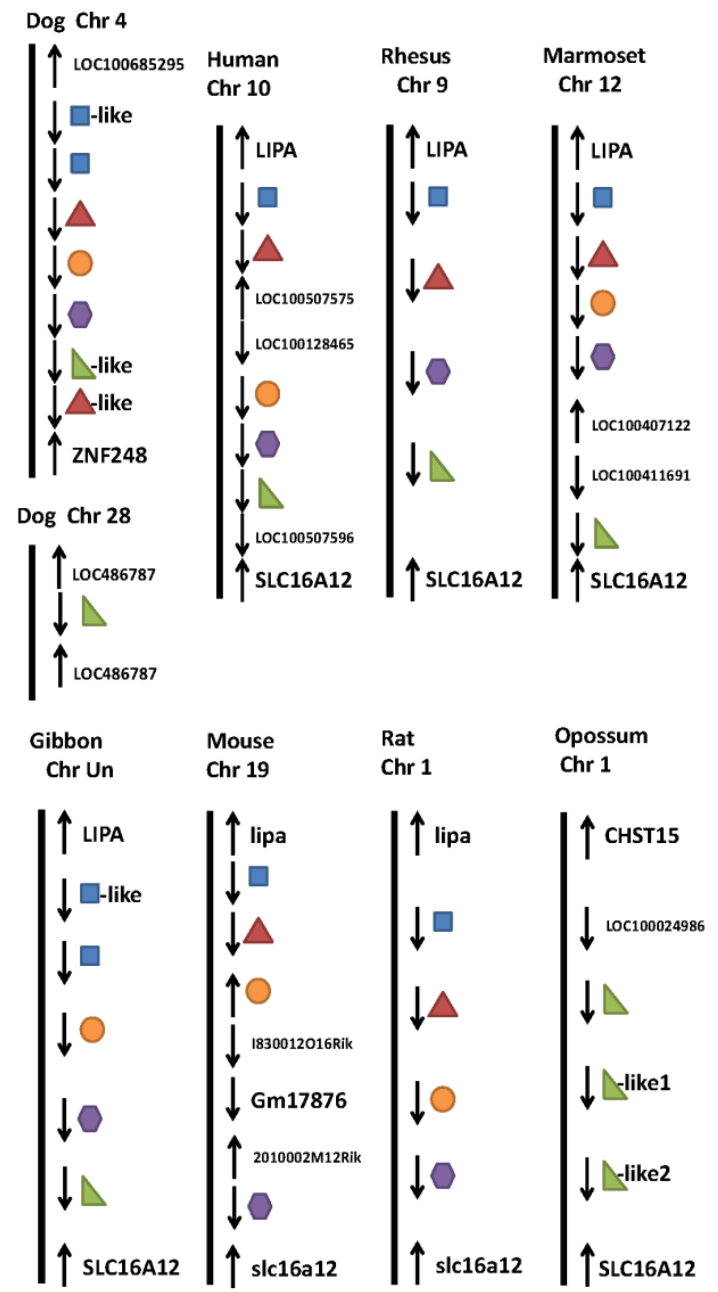

IFIT1 (also known as ISG56), IFIT2 (known as ISG54), IFIT3 (known as ISG60), and IFIT5 (known as ISG58) (Figure 1). However, IFIT3 is absent in gibbons, while IFIT5 does not exist in mice and rats. Opossums, chickens, frogs, and zebrafish possess IFIT5 only. In addition to these four members, humans, marmosets, orangutans, dogs, gibbons, mice and rats have an IFIT1-like (IFIT1L) gene, while dogs and mice have an IFIT3L gene. Moreover, opossums, chickens, frogs, and zebrafish have multiple IFIT5L genes (Figure 1). Several IFIT-related pseudogenes were also identified in human (IFIT6P), orangutan (IFIT1LP and IFIT2LP), dog (IFIT2LP and IFIT5LP), pig (IFIT3LP), cattle, horse and gibbon (IFIT2LP), and frog and zebrafish (IFIT5LP), respectively. Phylogenetic relationships of IFIT family genes are complicated (Figure 2). In most cases, members 2,3 and 5 are relatively close to each other, members 1 and $1 \mathrm{~L}$ are clustered together, and IFIT5L genes are near one another. These data show that re-annotation of the gene family among different species is needed.
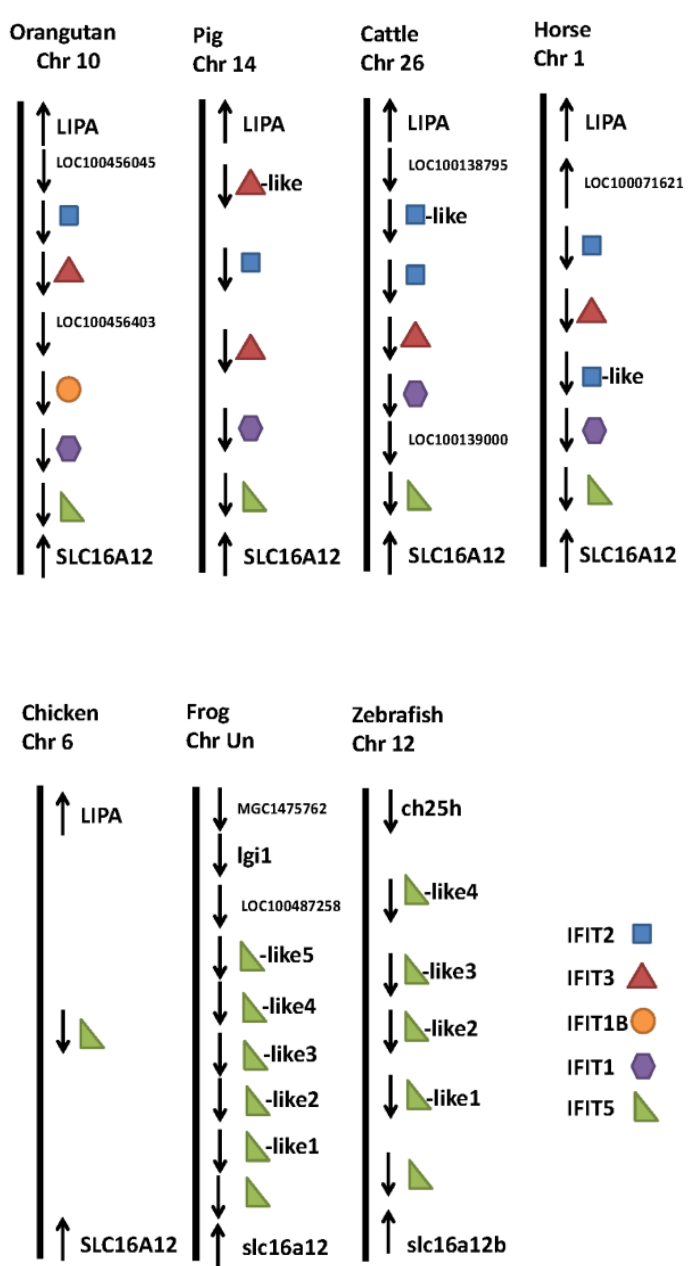

Figure I. Genomic neighborhood surrounding the IFIT family duplicated genes. The relative locations and orientations of both IFIT family genes and their adjacent neighbor genes were collected from the NCBI database plus chromosome number if available. 


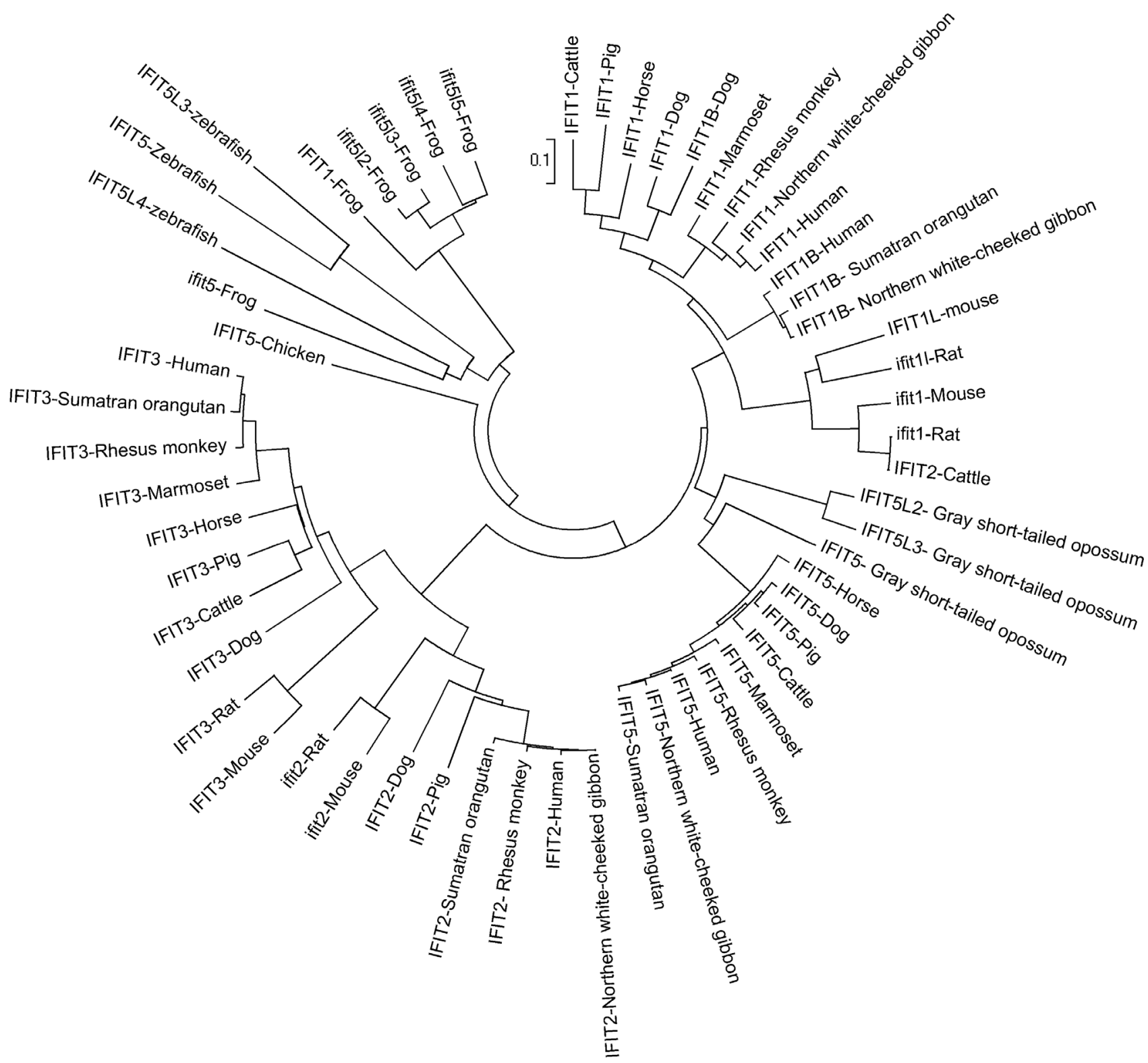

Figure 2. Phylogeny of IFIT family proteins. A neighbor-joining tree of IFIT family proteins was generated by MEGA4.0 [62].

The IFIT family is clustered on chromosomes 10 in human, 9 in rhesus, 12 in marmoset, 10 in orangutan, 14 in pig, 26 in cattle, 1 in horse, 19 in mouse, 1 in rat, 1 in opossum, 6 in chicken and 12 in zebrafish, but has not been placed on chromosomes in gibbon and frog. However, the IFIT family in the dog genome is split between two chromosomes: IFIT1, IFIT2 and IFIT3 on 4 and IFIT5 on 28 (Figure 1). Among these fifteen species, only three species - dog, opossum and frog do not have the family confined in a region between LIPA (lipase A, lysosomal acid, cholesterol esterase) and SLC16A12 (solute carrier family 16, member 12 (monocarboxylic acid transporter 12)) (Figure 1). However, IFIT5 is always located adjacent to SLC16A12, regardless of species (Figure 1). Most of the IFIT family genes have two exons and contain two or three IFN-stimulated response elements (IRSE) in their promoter regions [7]. The IRSE are important cis-acting elements recognized by IFN-stimulated gene factor 3 (ISGF3) that are activated by IFN and various stimuli [8].

\section{IFIT family expressions and cellular loca- tions}

Generally speaking, IFIT family genes are usually less abundantly expressed in the absence of stimuli. They are prominently induced by type I and type III interferons, especially IFN- $\alpha / \beta$ [9]. Various 
pathogens, particularly viruses, induce IFIT family gene expression. Both DNA- and RNA- viruses efficiently elicit IFIT family genes transcription. Cytomegalovirus (CMV) is a DNA virus, which broadly infects human and animals. IFIT2 was induced at 8 hours after infection with human CMV (HCMV)[10]. Adenovirus is a double-stranded linear DNA virus that causes upper respiratory infection in children. Zhao and colleagues [11] found that IFIT1 and IFIT2 were activated during the late stage of adenovirus type 12 infection in primary human fibroblasts. Other DNA viruses, like Simian virus 40 (SV40), a polyomavirus also stimulate IFIT family genes expression [12-13].

West Nile virus (WNV) is a positive-sense, single-stranded RNA virus with an extensive tropism that infects a broad number of species. In mouse, Ifit1 and Ifit2 are often induced after WNV infection [14]. Porcine reproductive and respiratory syndrome virus (PRRSV), an arterivirus that causes disease in all ages of swine, activates IFIT1 and IFIT3 expression in porcine alveolar macrophages (PAM), and IFIT1 and IFIT5 expression in lung [15-16]. Hantaviruses are negative-sense RNA viruses and include Hantaan virus (HTNV), Prospect Hill virus (PHV), Tula virus (TULV) and others. PHV and TULV infect human endothelial cells, resulting in strong induction of IFIT3 expression [17]. The influenza A virus is a negative-sense, single-stranded RNA virus. The IFIT family proteins, IFIT1, IFIT2 and IFTI3 were up-regulated in human primary macrophages in response to influenza virus infection [18] and IFIT2 is strongly up-regulated in peripheral blood of pediatric patients during the acute stage of influenza infection [19]. The only IFIT family gene in birds, IFIT5, was significantly increased in duck lung at day 1 post-infection with highly pathogenic influenza A virus (VN1203) as compared with low pathogenic influenza A virus (BC500) [20]. Other RNA viruses, such as Japanese encephalitis virus (JEV), lymphocytic choriomeningitis virus (LCMV), and rabies viruses also induce IFIT family genes expression [14, 21-22].

Lipopolysaccharide (LPS) of bacteria is an important agent that stimulates IFIT family genes expression. IFIT1, IFIT2, IFIT3 and IFIT5 are transcribed when human moncytes are infected with wild-type Neisseria meningitidis compared with LPS-deficient Neisseria meningitidis. Stimulation of Raw246.7 macrophages with LPS also elicited IFIT2 in a type I interferon dependent manner [23-24]. Chlamydia is another type of pathogen that activates IFIT gene family expression. During secondary infection of Chlamydia pneumonia in mouse mononuclear cells, Ifit1 and Ifit3 were up-regulated [25].
Protein functioning depends mainly on their subcellular locations. Generally speaking, IFIT family proteins function in the cytoplasm. IFIT1 is located in the cytosol. However, $\mathrm{Li}$ and co-workers found that IFIT1 interacted with mitochondrial membrane protein MAV1, indicating that IFIT1 is also located in mitochondria to regulate immune response [26]. Like IFIT1, IFIT3 is also located in the cytoplasm and mitochondria [27]. In addition to cytoplasm and mitochondria, IFIT2 also appears in microtubules. IFIT2 interacts with the cytoskeleton, which may play an important role in cell proliferation and microtubule dynamics [21]. IFIT5 is reported to reside in cytoplasm, but it does not interact with other IFIT family proteins [28].

\section{Signaling associated with IFIT family member expression}

IFIT family gene expression relies on pattern recognition and the JAK-STAT pathway. Pathogen-associated molecular patterns (PAMPs) are molecules associated with groups of pathogens including viruses, bacteria, fungi and others. Pattern recognition receptors (PRRs) recognize different PAMPs during pathogen infection and activate downstream signaling molecules [29]. As a result, Toll-like receptors (TLRs) signaling and RIG-like receptors (RLRs) signaling induce IFIT family gene expression [30]. TLR3 senses double-stranded RNA (dsRNA), TLR7 and TLR8 sense single-stranded RNA (ssRNA), and TLR9 recognizes CpG-DNA [31]. The TIR domain-containing adaptor inducing IFN- $\beta$ (TRIF)-dependent signaling pathway or myeloid differentiation primary response gene (88) (MyD88)-dependent signaling pathway transfect the signal from the TLRs, which leads to the activation of IRF3 or IRF7 by phosphorylation. The activated IRF3 or IRF7 is then translocated to the nucleus, resulting in type I interferon gene expression (Figure 3) [32]. For example, irf3(-/-) mice lack expression of type I interferon and IFIT family genes in macrophages and cortical neurons during WNV infection [33]. In addition, secretory IFNs bind to IFN receptors at the cell surface, which then activate Janus kinase (JAK) and signal transducers and activators of transcription (STAT) pathways. The phosphorylated STAT1, STAT2, and IRF9 form the ISGF3 complex that translocates into the nucleus and binds to the ISRE elements in the promoter of IFIT family genes, thus stimulating IFIT family genes expression (Figure 3) [34]. RIG-I-like receptors (RLRs) are located in the cytoplasm and recognize dsRNA that originated from the genomic RNA of dsRNA viruses or is generated during replication of ssRNA viruses [35]. The adaptor 
IFN- $\beta$-promoter stimulator 1 (IPS-1) located in mitochondria interacts with the caspase-recruitment domain (CARDs) of RLRs and triggers signaling cascades and enhances IFN expression, thus stimulating IFIT family gene expression though JAK-STAT signaling as a result of TLRs signaling (Figure 3) [36] .

\section{Antivirus properties and immune regula- tion of IFIT family}

IFIT family proteins are involved in many processes in response to viral infection, mainly by reducing virus replication. The IFIT family proteins contain a TPR (tetratricopeptide repeat) domain, a 34 amino acid motif folding in to a helix-turn-helix structure, which mediates protein interactions [4]. IFIT1 and IFIT2 are involved in a nonspecific antiviral program through their direct interactions with eIF3, which subsequently suppresses more than $60 \%$ of translation in cells and viruses during protein synthesis (Figure 4) [37-39]. The IFIT family, especially IFIT1 and IFIT3, restrict DNA and RNA virus replication, such as hepatitis B virus (HBV), human papillomavirus (HPV), hepatitis $\mathrm{C}$ virus $(\mathrm{HCV})$, West Nile virus (WNV) and others [13, 17, 28, 40-41]. Knock- down of IFIT1 though RNA interference in human hepatocytes enhanced HCV replication during infection [40]. Similar results were also observed in other IFIT family members during other viral infections [28]. Although, IFIT family genes commonly restrict virus replication through alternation of protein synthesis, more mechanisms need to be explored. An intriguing, newly found antiviral mechanism of IFIT family genes is the ability of IFIT family proteins to directly bind viral RNA. Viral RNA that carries 5'PPP-RNA is recognized by IFIT1, followed by sequestering with the IFIT complex that contains IFIT1, IFIT2 and IFIT3. IFIT5, which shares the highest sequence homology with IFIT1, is also associated with PPP-RNA, but has little interaction with IFIT2 and IFIT3 (Figure 4) [28]. These data provide evidence that the IFIT family members play an important role in killing invasive RNA. Working together with other ISGs, they are able to restrict virus replication. In addition, IFIT2 may limit replication of vesicular stomatitis virus (VSV) in brain. Virus titer was higher in ifit2 (-/-) mice compared to wild-type mice during VSV infection. However, ifit1 could not prevent VSV replication [42].

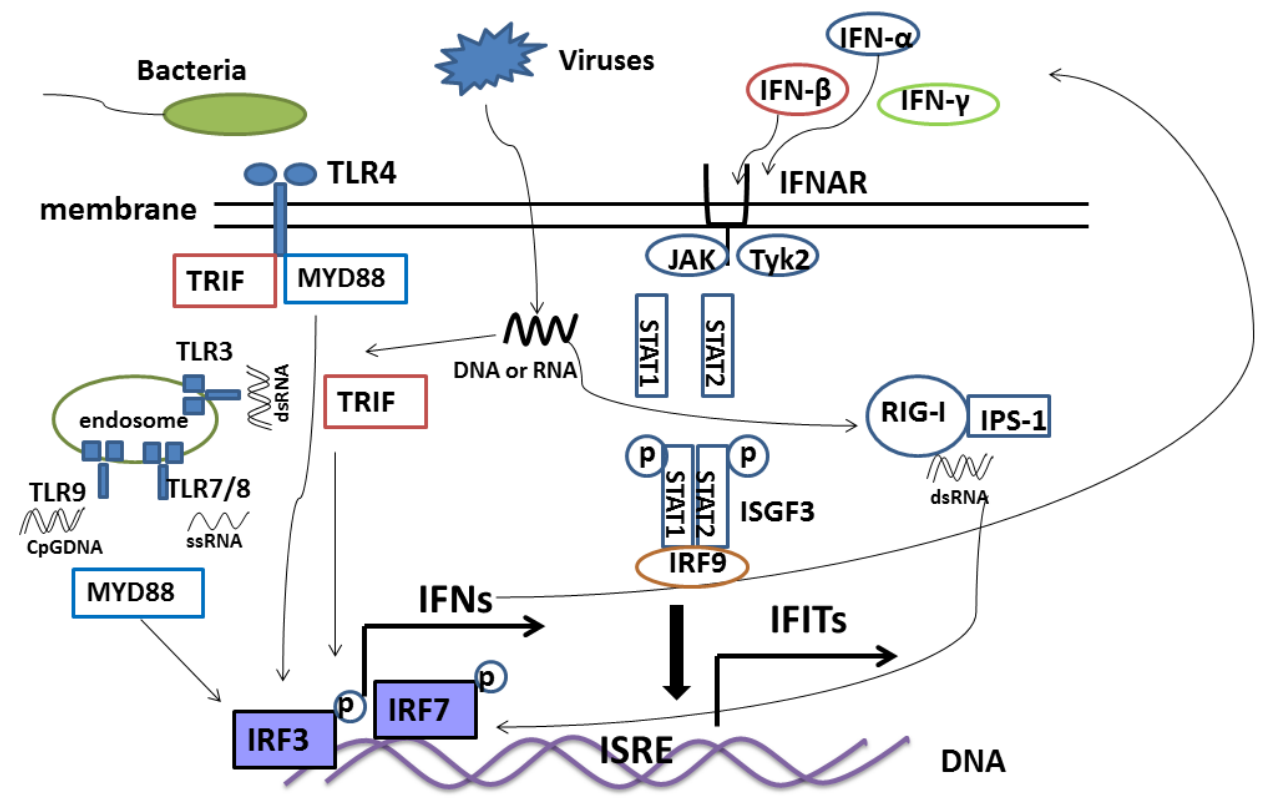

Figure 3. Signaling pathway of IFIT family genes. Toll-like receptors (TLRs) and RIG-like receptors (RLRs) are pattern recognition receptors (PRRs) families that recognize pathogen-associated molecular patterns (PAMPs) that trigger signaling. TLR3 and RIG-I sense dsRNA, while TLR4 senses LPS, TLR7/8 senses ssRNA and TLR9 senses CPG DNA. Adapter proteins MYD88, TRIF and ISP-I are used by the receptor complex that activate IRF3 and IRF7 by phosphorylaton, which then bind the DNA to stimulate IFN expression. Secreted IFN binds the receptor IFNAR at the cell surface, followed by activation of STATI and STAT2. The phosphorylated STATI, STAT2, and IRF9 form the ISGF3 complex, which is translocated into the nucleus, binds with the ISRE elements in the promoter of IFIT family genes, and thus stimulates IFIT family genes expression. 


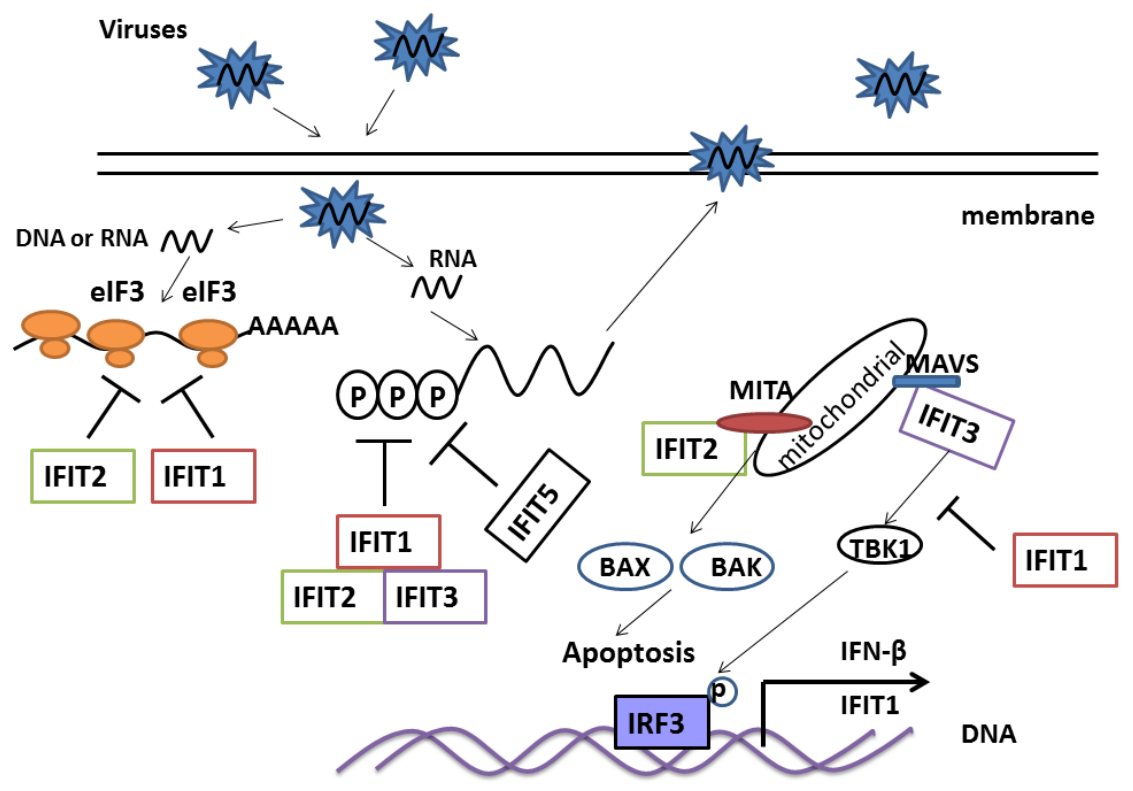

Figure 4. Antiviral and immune regulated function of IFIT family genes. IFITI and IFIT2 directly bind elF3 and suppress transcription of virus genes. IFITI, IFIT2 and IFIT3 form a complex in cytoplasm that recognizes and kills PPP-RNA. IFIT5 may also kill PPP-RNA directly. IFITI disrupts the interaction of MITA, MAVS and TBKI, which then negatively regulates the cellular antiviral response. IFIT2 interacts with MITA, and induces apoptosis via the mitochondrial pathway that is induced by the innate immune response. IFIT3 bridges TBKI to MAVS in mitochondria, which synergizes the activation of IRF3 and NF-KB to activate the immune response.

Innate immunity is the first line of defense against invading pathogens. The IFIT family shows potent antiviral ability so it is conceivable that they also influence the innate immune response. IFIT1 negatively regulates cellular antiviral response by disrupting the interaction of the MITA (mediator of IRF3 activation), MAVS (mitochondrial antiviral signaling protein) and TBK1 (TANK-binding kinase 1), which transfer signaling from RLRs recognized pathogens. Over-expression of IFIT1 could inhibit virus triggered activation of IFN- $\beta, N F-\kappa B$ and IRF3 (Figure 4) [26]. Furthermore, IFIT3, a new component of the MAVS complex located in mitochondria, could bridge TBK1 to MAVS on the mitochondrion which synergizes the activation of IRF3 and NF-KB (Figure 4) [27]. In addition, IFIT2 is also a MITA-associated protein, and induces apoptosis via the mitochondrial pathway that is induced by innate immune response. IFIT3 could block apoptosis by binding IFIT2 (Figure 4) [43].

\section{Virus inference with host IFIT family members}

Although the host immune response shows powerful antiviral capacity, viruses have evolved many processes to escape the host immune system, including inhibition of humoral response, interference with interferons and inhibition of cytokines and chemokines, for example [44]. When human hepatocytes were pretreated with IFN- $\alpha$ and then infected with HCV, IFN-induced IFIT1 expression was inhibited [40]. Other researchers also confirmed that HCV infection blocks the ISGs or cytokine expression, resulting in persistent HCV infection [45]. Varicella-zoster virus (VZV) may down-regulate IFIT1 and IFIT2 mRNA expression through its immediate-early protein ORF61, which antagonizes the IFN-beta pathway [46]. The NSP1 $\beta$ (Nonstructural Protein $1 \beta$ ) of PRRSV inhibits IFIT family gene expression by blocking nuclear translocation of STAT1 [47]. The 42-residue C-terminal of the Tula virus $\mathrm{Gn}$ reduces IFIT1 expression via unique interaction with TBK1 complex when TULV infects endothelial cells [17].

An interesting, newly identified strategy that viruses use to escape the antiviral activity of IFIT family genes is through $2^{\prime}-O$ methylation of the $5^{\prime}$ cap of viral RNA [48]. For example, C57BL/ 6 mice that were infected with the WNV mutant (WNV-E218A) strain that lacks 2'-O MTase showed $0 \%$ mortality compared with $40 \%$ mortality when mice were infected with wild-type WNV. Studies have shown that IFIT1 and IFIT2 play an important role in restricting infection of WNV lacking 2'-O methylation. In fact, transgenic expression of IFIT1 and IFIT2 in 3T3 cells strongly blocked the viruses which lacked 2'-O methylation [48]. Methylation in the $5^{\prime}$ cap structure of 
RNA is considered as an essential process for RNA translation and stability [49], therefore, 2'-O methylation of the viral genome that imitates host mRNA greatly benefits its life cycle and survival.

\section{Genetic variants of IFIT family genes}

Variants in ISGs and interferon pathway genes (IPGs) are usually associated with many immune traits, such as blood parameters, antibody titer and total white blood cells (WBC) [50]. Immune traits provide measurements of individual immune capacity. No doubt, variants in ISGs and IPGs could also influence host response to various pathogens. Recent reports showed that polymorphisms in IPGs and ISGs influence the effect of therapy against HCV infection. A tag SNP (rs2278034) in intron 11 of $A C K 1$ was associated with IFN therapy outcome in patients infected with HCV. SNP (rs8099917) in IL-28 influences its expression in patients, which interferes with drug therapy against HCV [51-52]. On the other hand, IFIT family genes are important ISGs, which play important roles in resisting $\mathrm{HCV}$ infection. The polymorphism (rs3004479) in the IFIT1 gene is strongly associated with sustained virological response (SVR) in HCV-1 patients [53]. SVR is a clinical index measuring detectable $\mathrm{HCV}$ in patient blood six months after treatment with type I interferon and ribavirin [54]. Patients with the A/A genotype have higher SVR than those with the $G / G$ genotype $(P<0.05)$, and achieve a better therapy outcome [53].

Certainly genetic polymorphisms can be used to improve innate resistance to virus infection in livestock species. Infectious disease often causes economic loss in animal production. In 2006, an outbreak of highly pathogenic porcine reproductive and respiratory syndrome (HP-PRRS) affected more than 2 million pigs and decimated the Chinese swine industry [55]. Millions of chickens are slaughtered or die every year because of Marek's disease (MD), Avian influenza (AI) and other infectious diseases [56-57]. Genetic selection for disease resistance may improve the ability of animals to respond to disease challenge [58]. An increasing number of SNPs in innate immune response related-genes, such as TLRs genes, are associated with infectious disease susceptibility [59-61]. IFIT family genes contain many variants (Table 1). So this family should have great potential to be probed for improved resistance to infectious diseases.

\section{Summary and perspectives}

Innate immunity is the first line of defense against invading pathogens. As IFIT family genes are involved in regulating innate immune responses, they are important targets with potent antiviral activities.
They could restrict various viruses, stimulate apoptosis, and regulate immune responses. Variants in IFIT family genes could influence therapy for infectious diseases. However, many questions remain. What stimulated the members of this clustered gene family to be duplicated during evolution? How have the genomic structures of duplicated genes diverged and how have structural divergences among the family members contributed to functional diversities associated with innate resistance to virus infection? These questions need to be explored further. We believe that additional understanding of the molecular and functional diversities of the IFIT members is imperative for developing more effective vaccines and inventing novel intervention strategies to combat viral outbreaks in both humans and animals.

Table I. Numbers of SNPs in the IFITs family genes currently available in NCBI dbSNP Database for five species

\begin{tabular}{lllll}
\hline Species & IFIT1 & IFIT2 & IFIT3 & IFIT5 \\
\hline Human & 267 & 151 & 351 & 210 \\
Mouse & 170 & 469 & 87 & No \\
Dog & 7 & 13 & 2 & 77 \\
Cattle & 16 & 44 & 89 & 38 \\
Pig & 2 & 2 & 4 & 2 \\
\hline
\end{tabular}

\section{Competing Interests}

The authors have declared that no competing interest exists.

\section{Acknowledgements}

This work was supported by the National Pork Board, USDA ARS and PRRS CAP, USDA NIFA Award 2008-55620-19132. Mr. Xiang Zhou is recipient of the China Scholarship Council assistantship for a joint Ph.D. program between Washington State University, USA and Huazhong Agricultural University, China.

\section{References}

1. de Weerd NA, Samarajiwa SA, Hertzog PJ. Type I interferon receptors: biochemistry and biological functions. J Biol Chem. 2007; 282: 20053-7.

2. Fensterl V, Sen GC. Interferons and viral infections. Biofactors. 2009; 35: 14-20.

3. de Veer MJ, Holko M, Frevel M, Walker E, Der S, Paranjape JM, et al. Functional classification of interferon-stimulated genes identified using microarrays. J Leukoc Biol. 2001; 69: 912-20.

4. D'Andrea LD, Regan L. TPR proteins: the versatile helix. Trends Biochem Sci. 2003; 28: 655-62.

5. Chebath J, Merlin G, Metz R, Benech P, Revel M. Interferon-induced 56,000 Mr protein and its mRNA in human cells: molecular cloning and partial sequence of the cDNA. Nucleic Acids Res. 1983; 11: 1213-26. 
6. Kusari J, Sen GC. Transcriptional analyses of interferon-inducible mRNAs. Mol Cell Biol. 1987; 7: 528-31.

7. Fensterl V, Sen GC. The ISG56/IFIT1 gene family. J Interferon Cytokine Res. 2011; 31: 71-8.

8. Gobin SJ, Peijnenburg A, Keijsers V, van den Elsen PJ. Site alpha is crucial for two routes of IFN gamma-induced MHC class I transactivation: the ISRE-mediated route and a novel pathway involving CIITA. Immunity. 1997; 6: 601-11.

9. Der SD, Zhou A, Williams BR, Silverman RH. Identification of genes differentially regulated by interferon alpha, beta, or gamma using oligonucleotide arrays. Proc Natl Acad Sci U S A. 1998; 95: 15623-8.

10. Zhu H, Cong JP, Shenk T. Use of differential display analysis to assess the effect of human cytomegalovirus infection on the accumulation of cellular RNAs: induction of interferon-responsive RNAs. Proc Natl Acad Sci U S A. 1997; 94: 13985-90.

11. Zhao H, Boije H, Granberg F, Pettersson U, Svensson C. Activation of the interferon-induced STAT pathway during an adenovirus type 12 infection. Virology. 2009; 392: 186-95.

12. Grinde B, Gayorfar M, Rinaldo CH. Impact of a polyomavirus (BKV) infection on mRNA expression in human endothelial cells. Virus Res. 2007; 123: 86-94.

13. Rathi AV, Cantalupo PG, Sarkar SN, Pipas JM. Induction of interferon-stimulated genes by Simian virus $40 \mathrm{~T}$ antigens. Virology. 2010; 406: 202-11

14. Wacher C, Muller M, Hofer MJ, Getts DR, Zabaras R, Ousman SS, et al. Coordinated regulation and widespread cellular expression of interferon-stimulated genes (ISG) ISG-49, ISG-54, and ISG-56 in the central nervous system after infection with distinct viruses. J Virol. 2007; 81: 860-71.

15. Xiao S, Mo D, Wang Q, Jia J, Qin L, Yu X, et al. Aberrant host immune response induced by highly virulent PRRSV identified by digital gene expression tag profiling. BMC Genomics. 2010; 11: 544.

16. Zhou $\mathrm{P}$, Zhai $\mathrm{S}$, Zhou $\mathrm{X}$, Lin $\mathrm{P}$, Jiang $\mathrm{T}, \mathrm{Hu} \mathrm{X}$, et al. Molecular characterization of transcriptome-wide interactions between highly pathogenic porcine reproductive and respiratory syndrome virus and porcine alveolar macrophages in vivo. Int J Biol Sci. 2011; 7: 947-59.

17. Matthys V, Gorbunova EE, Gavrilovskaya IN, Pepini T, Mackow ER. The C-terminal 42 residues of the Tula virus $\mathrm{Gn}$ protein regulate interferon induction. J Virol. 2011; 85: 4752-60.

18. Lietzen N, Ohman T, Rintahaka J, Julkunen I, Aittokallio T, Matikainen $\mathrm{S}$, et al. Quantitative subcellular proteome and secretome profiling of influenza A virus-infected human primary macrophages. PLoS Pathog. 2011; 7: e1001340.

19. Kawada J, Kimura H, Kamachi $Y$, Nishikawa K, Taniguchi M, Nagaoka $\mathrm{K}$, et al. Analysis of gene-expression profiles by oligonucleotide microarray in children with influenza. J Gen Virol. 2006; 87: 1677-83.

20. Vanderven HA, Petkau K, Ryan-Jean KE, Aldridge JR, Jr., Webster RG, Magor KE. Avian influenza rapidly induces antiviral genes in duck lung and intestine. Mol Immunol. 2012; 51: 316-24.

21. Saha S, Sugumar P, Bhandari P, Rangarajan PN. Identification of Japanese encephalitis virus-inducible genes in mouse brain and characterization of GARG39/IFIT2 as a microtubule-associated protein. J Gen Virol. 2006; 87: 3285-9.

22. Saha S, Rangarajan PN. Common host genes are activated in mouse brain by Japanese encephalitis and rabies viruses. J Gen Virol. 2003; 84: 1729-35.

23. Ovstebo R, Olstad OK, Brusletto B, Moller AS, Aase A, Haug KB, et al. Identification of genes particularly sensitive to lipopolysaccharide (LPS) in human monocytes induced by wild-type versus LPS-deficient Neisseria meningitidis strains. Infect Immun. 2008; 76: 2685-95.

24. Berchtold S, Manncke B, Klenk J, Geisel J, Autenrieth IB, Bohn E. Forced IFIT-2 expression represses LPS induced TNF-alpha expression at posttranscriptional levels. BMC Immunol. 2008; 9: 75.

25. Kylaniemi MK, Haveri A, Vuola JM, Puolakkainen M, Lahesmaa R. Gene expression signatures characterizing the development of lymphocyte response during experimental Chlamydia pneumoniae infection. Microb Pathog. 2009; 46: 235-42.

26. Li Y, Li C, Xue P, Zhong B, Mao AP, Ran Y, et al. ISG56 is a negative-feedback regulator of virus-triggered signaling and cellular antiviral response. Proc Natl Acad Sci U S A. 2009; 106: 7945-50.

27. Liu XY, Chen W, Wei B, Shan YF, Wang C. IFN-induced TPR protein IFIT3 potentiates antiviral signaling by bridging MAVS and TBK1. J Immunol. 2011; 187: 2559-68.

28. Pichlmair A, Lassnig C, Eberle CA, Gorna MW, Baumann CL, Burkard TR, et al. IFIT1 is an antiviral protein that recognizes 5'-triphosphate RNA. Nat Immunol. 2011; 12: 624-30.
29. Takeuchi O, Akira S. Pattern recognition receptors and inflammation. Cell. 2010; 140: 805-20.

30. Kawai T, Akira S. The role of pattern-recognition receptors in innate immunity: update on Toll-like receptors. Nat Immunol. 2010; 11: 373-84.

31. O'Neill LA, Bowie AGz. The family of five: TIR-domain-containing adaptors in Toll-like receptor signalling. Nat Rev Immunol. 2007; 7: 353-64.

32. Negishi H, Fujita $Y$, Yanai H, Sakaguchi S, Ouyang X, Shinohara M, et al. Evidence for licensing of IFN-gamma-induced IFN regulatory factor 1 transcription factor by MyD88 in Toll-like receptor-dependent gene induction program. Proc Natl Acad Sci U S A. 2006; 103: 15136-41.

33. Daffis S, Samuel MA, Keller BC, Gale M, Jr., Diamond MS. Cell-specific IRF-3 responses protect against West Nile virus infection by interferon-dependent and -independent mechanisms. PLoS Pathog. 2007; 3: e106.

34. Elco CP, Sen GC. Stat1 required for interferon-inducible but not constitutive responsiveness to extracellular dsRNA. J Interferon Cytokine Res. 2007; 27: 411-24.

35. Zhou X, Jiang T, Du X, Zhou P, Jiang Z, Michal JJ, et al. Molecular characterization of porcine SARM1 and its role in regulating TLRs signaling during highly pathogenic porcine reproductive and respiratory syndrome virus infection in vivo. Dev Comp Immunol. 2012.

36. Kawai $\mathrm{T}$, Akira S. Innate immune recognition of viral infection. Nat Immunol. 2006; 7: 131-7.

37. Hui DJ, Terenzi F, Merrick WC, Sen GC. Mouse p56 blocks a distinct function of eukaryotic initiation factor 3 in translation initiation. J Biol Chem. 2005; 280: 3433-40.

38. Guo J, Hui DJ, Merrick WC, Sen GC. A new pathway of translational regulation mediated by eukaryotic initiation factor 3. EMBO J. 2000; 19: 6891-9.

39. Terenzi F, Hui DJ, Merrick WC, Sen GC. Distinct induction patterns and functions of two closely related interferon-inducible human genes, ISG54 and ISG56. J Biol Chem. 2006; 281: 34064-71.

40. Raychoudhuri A, Shrivastava S, Steele R, Kim H, Ray R, Ray RB. ISG56 and IFITM1 proteins inhibit hepatitis C virus replication. J Virol. 2011; 85: 12881-9.

41. Saikia P, Fensterl V, Sen GC. The inhibitory action of P56 on select functions of E1 mediates interferon's effect on human papillomavirus DNA replication. J Virol. 2010; 84: 13036-9.

42. Fensterl V, Wetzel JL, Ramachandran S, Ogino T, Stohlman SA, Bergmann CC, et al. Interferon-Induced Ifit2/ISG54 Protects Mice from Lethal VSV Neuropathogenesis. PLoS Pathog. 2012; 8: e1002712.

43. Stawowczyk M, Van Scoy S, Kumar KP, Reich NC. The interferon stimulated gene 54 promotes apoptosis. J Biol Chem. 2011; 286: 7257-66.

44. Alcami A, Koszinowski UH. Viral mechanisms of immune evasion. Immunol Today. 2000; 21: 447-55

45. Jouan L, Chatel-Chaix L, Melancon P, Rodrigue-Gervais IG, Raymond VA, Selliah S, et al. Targeted impairment of innate antiviral responses in the liver of chronic hepatitis C patients. J Hepatol. 2012; 56: 70-7.

46. Zhu H, Zheng C, Xing J, Wang S, Li S, Lin R, et al. Varicella-zoster virus immediate-early protein ORF61 abrogates the IRF3-mediated innate immune response through degradation of activated IRF3. J Virol. 2011; 85: $11079-89$.

47. Patel D, Nan Y, Shen M, Ritthipichai K, Zhu X, Zhang YJ. Porcine reproductive and respiratory syndrome virus inhibits type I interferon signaling by blocking STAT1/STAT2 nuclear translocation. J Virol. 2010; 84: 11045-55.

48. Daffis S, Szretter KJ, Schriewer J, Li J, Youn S, Errett J, et al. 2'-O methylation of the viral mRNA cap evades host restriction by IFIT family members. Nature. 2010; 468: 452-6.

49. Furuichi Y, Shatkin AJ. Viral and cellular mRNA capping: past and prospects. Adv Virus Res. 2000; 55: 135-84.

50. Flori L, Gao Y, Laloe D, Lemonnier G, Leplat JJ, Teillaud A, et al. Immunity traits in pigs: substantial genetic variation and limited covariation. PLoS One. 2011; 6: e22717.

51. Abe H, Hayes CN, Ochi H, Maekawa T, Tsuge M, Miki D, et al. IL28 variation affects expression of interferon stimulated genes and peg-interferon and ribavirin therapy. J Hepatol. 2011; 54: 1094-101.

52. Fujimoto $\mathrm{Y}$, Ochi $\mathrm{H}$, Maekawa $\mathrm{T}$, Abe $\mathrm{H}$, Hayes $\mathrm{CN}$, Kumada $\mathrm{H}$, et al. A single nucleotide polymorphism in activated $\mathrm{Cdc} 42$ associated tyrosine kinase 1 influences the interferon therapy in hepatitis $\mathrm{C}$ patients. J Hepatol. 2011; 54: 629-39.

53. Lopez-Rodriguez R, Trapero-Marugan $M$, Borque MJ, Roman M, Hernandez-Bartolome A, Rodriguez-Munoz Y, et al. Genetic variants of interferon-stimulated genes and IL-28B as host prognostic factors of response to combination treatment for chronic hepatitis C. Clin Pharmacol Ther. 2011; 90: 712-21. 
54. Veldt BJ, Heathcote EJ, Wedemeyer H, Reichen J, Hofmann WP, Zeuzem $\mathrm{S}$, et al. Sustained virologic response and clinical outcomes in patients with chronic hepatitis C and advanced fibrosis. Ann Intern Med. 2007; 147: 677-84

55. Schoggins JW, Wilson SJ, Panis M, Murphy MY, Jones CT, Bieniasz P, et al. A diverse range of gene products are effectors of the type I interferon antiviral response. Nature. 2011; 472: 481-5.

56. Parvizi P, Abdul-Careem MF, Haq K, Thanthrige-Don N, Schat KA, Sharif S. Immune responses against Marek's disease virus. Anim Health Res Rev. 2010; 11: 123-34.

57. Pantin-Jackwood MJ, Swayne DE. Pathogenesis and pathobiology of avian influenza virus infection in birds. Rev Sci Tech. 2009; 28: 113-36.

58. Lunney JK, Chen H. Genetic control of host resistance to porcine reproductive and respiratory syndrome virus (PRRSV) infection. Virus Res. 2010; 154: 161-9.

59. Hong YH, Kim ES, Lillehoj HS, Lillehoj EP, Song KD. Association of resistance to avian coccidiosis with single nucleotide polymorphisms in the zyxin gene. Poult Sci. 2009; 88: 511-8.

60. White SN, Taylor KH, Abbey CA, Gill CA, Womack JE. Haplotype variation in bovine Toll-like receptor 4 and computational prediction of a positively selected ligand-binding domain. Proc Natl Acad Sci U S A. 2003; 100: 10364-9.

61. Uenishi H, Shinkai H, Morozumi T, Muneta Y, Jozaki K, Kojima-Shibata $\mathrm{C}$, et al. Polymorphisms in pattern recognition receptors and their relationship to infectious disease susceptibility in pigs. BMC Proc. 2011; 5 Suppl 4: S27.

62. Tamura K, Dudley J, Nei M, Kumar S. MEGA4: Molecular Evolutionary Genetics Analysis (MEGA) software version 4.0. Mol Biol Evol. 2007; 24: 1596-9. 